\title{
Application of photon Doppler velocimetry to direct impact Hopkinson pressure bars
}

\author{
Lewis J Lea ${ }^{1,}$ a) and Andrew P Jardine ${ }^{1}$ \\ SMF Fracture and Shock Physics Group, Cavendish Laboratory, JJ Thomson Avenue, Cambridge, CB3 OHE, \\ $U K$
}

(Dated: 11 January 2016)

Direct impact Hopkinson pressure bar systems offer many potential advantages over split Hopkinson pressure bars, including access to higher strain rates, higher strains for equivalent striker velocity and system length, lower dispersion and faster achievement of force equilibrium. Currently these advantages are gained at the expense of all information about the striker impacted specimen face, preventing the experimental determination of force equilibrium, and requiring approximations to be made on the sample deformation history. In this paper we discuss an experimental method and complementary data analysis for using Photon Doppler velocimetry to measure surface velocities of the striker and output bars in a direct impact bar experiment, allowing similar data to be recorded as in a split bar system. We discuss extracting velocity and force measurements, and the precision of measurements. Results obtained using the technique are compared to equivalent split bar tests, showing improved stress measurements for the lowest and highest strains in fully dense metals, and improvement for all strains in slow and non equilibrating materials.

\section{INTRODUCTION}

Two general types of Hopkinson Bar systems exist for compression experiments: Split Hopkinson Pressure Bars (SHPBs, also known as Kolsky Bars ${ }^{1,2}$ ) and Direct Impact systems $(\mathrm{DIHBs})^{3,4}$. In a typical SHPB test, a sample is placed between an input and output bar , depicted at the top of Figure 1. The input bar is struck by a striker bar, passing a pressure wave through the input bar, into the sample and out through the output bar. A fourth bar traps the momentum transferred by the striker, protecting the experiment. The local strain at some point on the input and output bars is typically measured using strain gauges. The bars remain elastic during the experiment, allowing the force and velocity histories of the end faces of the bars, and therefore the sample, to be found from contact measurements at a distance.

In direct impact bars, the input bar is removed and the sample is struck directly by the striker bar, shown at the bottom of Figure 1. Striking the sample directly has many benefits over using an input bar. Higher strains can be achieved with an equivalent system as the striker bar unloads over multiple passes of the wave, rather than the single one in an SHPB. Higher strain rates can be achieved as striking the sample directly bypasses the collision between the striker and input bar, which limits the firing velocity based on the properties of the bars ${ }^{4}$,

$$
V_{\text {max }}=\frac{2 Y_{b a r}}{Z_{b a r}}
$$

where $Y_{b a r}$ is the yield stress of the bars and $Z_{b a r}$ is their acoustic impedance. In a DIHB experiment the striker

a) $11379 @$ cam.ac.uk decelerates more slowly as it continues moving with the deforming sample, and does so for multiple passes of the wave, meaning the peak and average stresses experienced by the striker bar for a given velocity are lower, and hence higher shot velocities can be used given the same bar material.

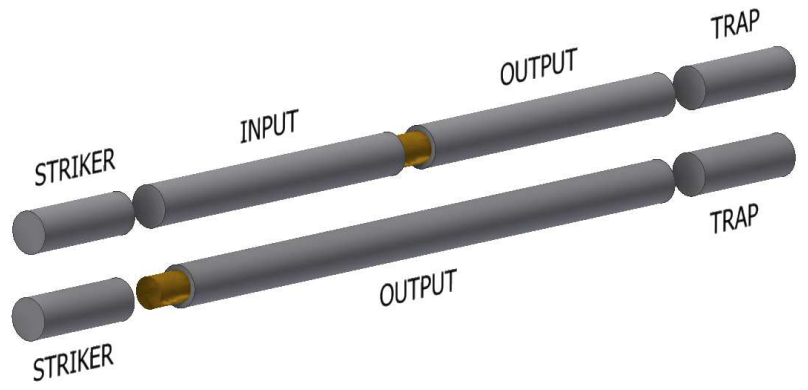

FIG. 1. The co-axial bar arrangements in typical SHPB (top) and DIHB (bottom) systems. The experiment is initiated by the striker bars impacting with the system.

The removal of the input bar substantially reduces the length of the system, making direct impact systems more convenient to use. The geometry also removes the Pochhammer-Chree dispersion that occurs as the pulse travels though the input bar ${ }^{6}$ to reach the sample. Some specimen types may reach force equilibrium faster in a DIHB setup as removing dispersion makes the rising edge of the stress pulse steeper, however equilibrium is still dominated by the properties of the specimen. Dispersion between the sample and measurement points is also reduced, however it cannot be removed entirely due to limitations set by Saint-Venant's principle ${ }^{5}$.

Currently, the advantages gained from a direct impact setup come at significant cost. Removing the input bar removes all gauge information about the input face, meaning approximations have to be made about the striker face velocity and therefore strain and strain rate. 
Similarly the achievement of stress equilibrium in a sample cannot be experimentally verified. Consequently, even in materials which equilibrate quickly (such as fully dense metals) DIHB data is generally less reliable than SHPB data. In materials slow to reach force equilibrium, and materials which undergo compaction such as foams or cellular materials, DIHB methods are often unusable.

Since the earliest direct impact experiments, high speed photography and streak methods have been available to measure the velocity of the striker bar ${ }^{7}$. However these methods provide no information about the force at the striker face, are of limited resolution and are time consuming. Progress has recently been made using strain gauges to instrument striker bars ${ }^{8}$. However strain gauges remain susceptible to damage even in specialised barrels. These methods make DIHB experiments much less convenient to perform, both during the experiment and in post-processing, sacrificing one of the main attractions of this technique.

Recently, Photon Doppler Velocimetry (PDV) methods have been developed by Avinadav et al. ${ }^{9}$ which provide equivalent optical measurements to those made by strain gauges on an SHPB. A non-contact measurement of the surface velocity of the bar is made by reflecting a laser beam off the surface. The source and returning light are combined, creating a time varying interference pattern from which the velocity history of a bar can be deduced. These methods measure local velocity, not strain, making them well suited to obtaining data from a striker bar during a direct impact experiment where there are velocity components due to bulk motion and elastic deformation.

In this paper, we demonstrate the use of PDV to measure both the velocity and force on the striker face of a direct impact bar specimen, allowing data to be collected that is similar to that obtained using an SHPB, with similar convenience. Internal reflections in the striker bar and methods for improving the convenience of and accuracy of all PDV-based bar systems are discussed. Results are compared with traditional direct impact analysis of the same data, and with SHPB tests under the same conditions for two fully dense metals, a metallic foam and a polymer.

\section{EXPERIMENTAL TECHNIQUES}

\section{A. The Bar Systems}

The DIHB system was used in a standard arrangement ${ }^{3}$, as shown in Figure 2. The bars were a Dural alloy, $25 \mathrm{~mm}$ in diameter, the output bar was $1 \mathrm{~m}$ long and the striker was $25 \mathrm{~cm}$ long. 12.5 $\mathrm{mm}$ Dural and magnesium systems were used for softer samples. The sample of interest was attached to the face of the output bar using Vaseline lubricant ${ }^{10}$.

The SHPB system consisted of $12.5 \mathrm{~mm}$ diameter bars, with $50 \mathrm{~cm}$ input and output bars, and a $25 \mathrm{~cm}$ striker bar. Vaseline was similarly used to provide an equivalent test. In both cases the bars were mounted coaxially on V-blocks and the striker was propelled using a gas gun. The striker velocity before impact was measured using a pair of light gates, the second of which was placed such that it was immediately next to the test sample and was and used to trigger the optical diagnostics.

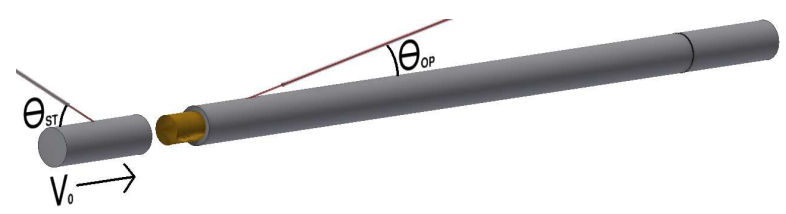

FIG. 2. An illustration of the DIHB experimental geometry. The incidence angles between the probing lasers and the bar axis in a DIHB system. The spot probing the striker bar is positioned based on the location of the bar at the moment of impact.

\section{B. Optical Velocity Measurements}

A $1.55 \mu m$ optical fibre interferometer ${ }^{11}$, shown schematically in Figure 3, was used to probe the bar surfaces. Light reflecting off of the bar surface is classically Doppler shifted by

$$
\Delta f=\frac{2 V}{\lambda} .
$$

Unshifted light is partially reflected back at the cleaved fibre end, and added to surface reflected light collected by the same fibre. The combination creates an interference pattern with beat frequency dependant on the shift. The entire pattern is modulated by a faster component averaged out during measurement. The resultant measured intensity profile is

$$
I(t)=I_{0}+I_{1} \cos (2 \pi \Delta f t)
$$

where $I_{0}$ and $I_{1}$ are dependent on many variables including the incident intensity of the laser and the reflectivity of the surface. However they are unimportant, as all required information is encoded in

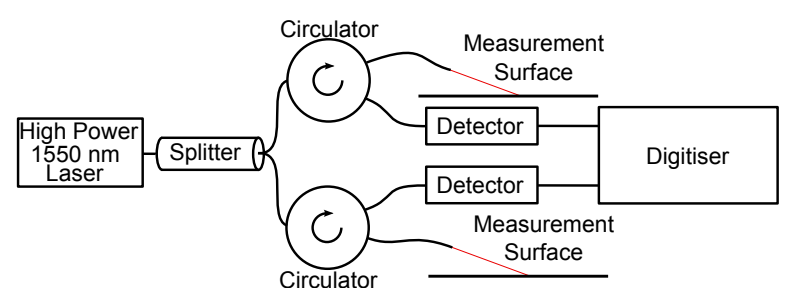

FIG. 3. Schematic of the photon Doppler interferometer: Light is passed through the circulator to the measurement surface, and then from the surface to $\mathrm{GHz}$ response photodiodes, which output an intensity proportional voltage. Interferometer probes were orientated as shown in Figure 2. 

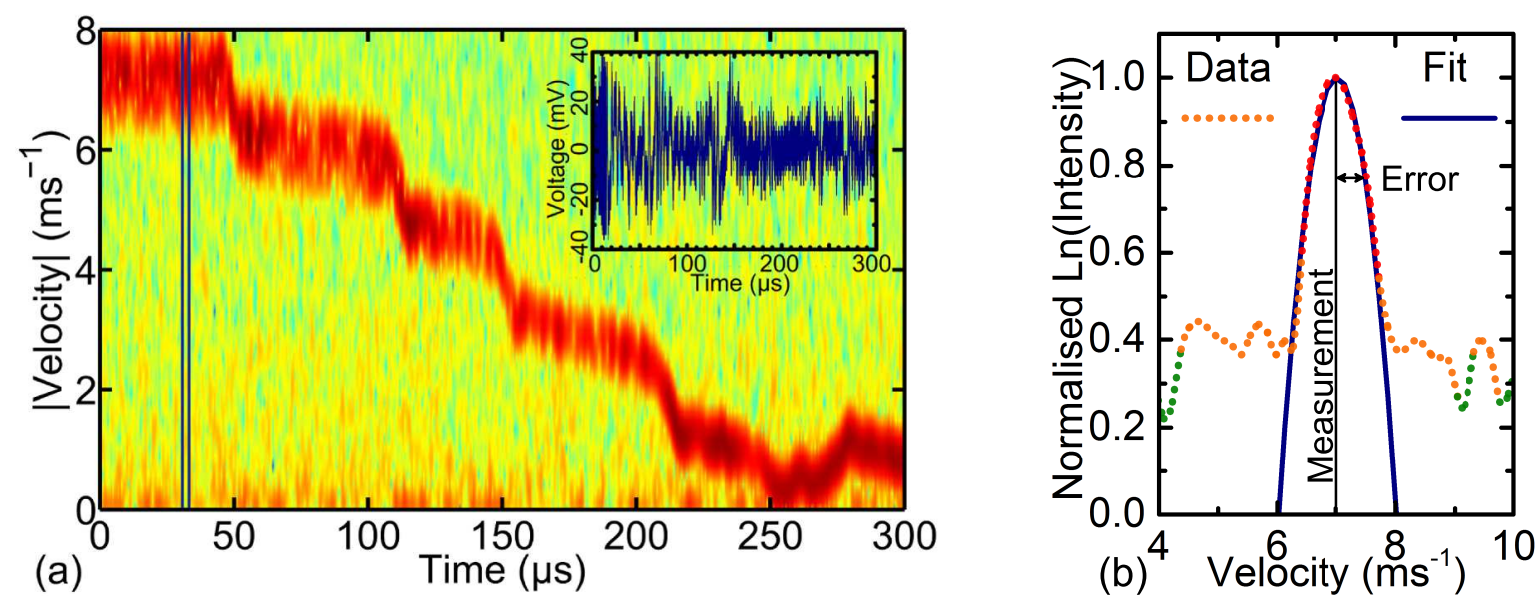

FIG. 4. (a) A map of the velocities obtained from Fourier transforming interference pattern, as described in the body text, for a typical DIHB experiment. The interference pattern which generated this trace is shown as an inset. The velocities depicted relate to the frequencies measured, as defined by equations 2 and 3. (b) The velocity and error at each time step is found by fitting a Gaussian curve to the spectrum (note that the vertical axis is logarithmic, so the Gaussian curve appears as a parabola).

the frequency of the pattern. Frequency measurement makes the technique resilient to typical laboratory noise.

Velocity histories of the faces of the striker bar and output bar in contact with the sample were deduced from surface velocity measurements made further down the bars as depicted in Figure 2. Surface velocities were extracted from the PDV data using a standard procedure $^{11}$. Each interference pattern, exemplified by the inset in Figure 4 (a) was split into a series of overlapping time windows. Each section was fast Fourier transformed, creating a series of spectra, depicted in Figure 4 (a), where each vertical slice is the spectrum of a single segment. An example of a single spectrum is shown in Figure 4 (b) compared to the marked region in Figure 4 (a). Velocities are obtained by fitting a Gaussian curve to the peak in each spectrum, as shown in Figure 4 (b). The centre value of the peak is taken as the velocity, and its standard deviation as the error.

A strong signal near zero velocity, visible in Figure 4 (a), caused by imperfections in the optical components is both subtracted and avoided during fitting. Interpolation is used to fit a smooth curve between the velocity measurement points. Window lengths of $8.2 \mu$ s were used with a $7.2 \mu$ s overlap, giving a $2.1 \mu$ s step size.

True velocities are found by accounting for the projection of the bar axis onto the laser axis

$$
V_{\text {true }}=\frac{V_{\text {meas }}}{\cos \theta}
$$

where $\theta$ is incidence angle of the probing laser depicted in Figure 2. Light gate measurements were used to confirm the measurement of striker velocity immediately before impact, which is required for correct analysis as shown in section III.

\section{Input \& Output Bar Measurements}

The strain gauges usually placed on the input and output bars were replaced with retro-reflective paint, which consists of a layer of small glass spheres that cause incident laser light to reflect back on itself. Retro reflection allowed the fibres to be placed at glancing angles (typically $10^{\circ}$ ) with reference to the bar surface without the severe loss of backscattered light that normally results from diffuse reflection. Glancing angle measurements lead to a reduction in errors caused by the precision of the laser incidence angle and bending velocities during the experiment.

Errors associated with the bending of the bars were quantified by pointing fibres at opposite sides of the same point on the bar, allowing the measurement projections to be separated into axial and radial (bending) velocities ${ }^{12}$. Bending modes cause a velocity towards one probe, and way from the other, resulting in the measured velocities

$$
V_{1}=V_{\text {axial }} \cos \theta+V_{\text {bend }} \sin \theta,
$$

and

$$
V_{2}=V_{\text {axial }} \cos \theta-V_{\text {bend }} \sin \theta,
$$

allowing for the separation of the axial and bending velocities. The trace from a single probe can then be compared with the pure axial velocity extracted from a double probe measurement. At a fibre incident angle, $\theta$, of $10^{\circ}$, and alignment of the bars such that the input bar can be slid into the gun barrel from its mounts, the $\sin \theta$ projection of bending velocities reduced the related errors below $1 \%$ when using $12.7 \mathrm{~mm}$ bars of the SHPB set-up. This error is further decreased in a DIHB set-up as the maximum radii of the forces from the cylindrical axis are 
restricted to that of the sample, smaller than that in the striker-input bar collision in the SHPB. The reduction in the projection of bending modes allowed each bar to be probed with a single laser, while still maintaining better than $1 \%$ accuracy. Hence reducing the number of velocimeter channels required from four to two.

Measurements are carried out at points along the bar surface, as depicted in Figure 2, and are time shifted back to the bar faces. Probe positions were chosen to be at least 5 bar radii from the contact face, to satisfy Saint-Venant's principle ${ }^{5}$. The positioning ensured the stresses were uniform across the entire cross section of the bar, making surface measurements representative of the bulk.

Applying the laser probe at glancing incidence causes the spot size of the laser on the bar to increase, and therefore the time resolution of the probe to decrease. The spot size at the surface is of the order of a millimetre in diameter, similar to the size of a semiconductor gauge. Given a typical bar sound speed of over $1 \mathrm{~km} \mathrm{~s}^{-1}$ temporal resolution remains better than $1 \mu \mathrm{s}$.

\section{Striker Bar Measurements}

As the striker bar is repeatedly fired from the barrel, it is not coated in any kind of reflective or retro reflective paint. Instead, the incident laser was shone at a steeper angle (typically $30^{\circ}$ ) to increase the diffuse return. Despite the absence of any paint, a signal was still collectable with the fibre placed $5 \mathrm{~mm}$ or closer to the bar surface. The increase in projection angle had no large effect on the influence of bending modes which are still negligibly small due to the reduced moments in direct impact, as discussed in section II C.

The laser probe was placed at the mid point of the bar, $12.5 \mathrm{~cm}$ from the sample, firstly to satisfy Saint-Venant's principle as discussed above but also to aid in and properly visualise wave separation, discussed in section III A. The probe can be placed anywhere further than the Saint-Venant's limit, including the back face. The location of this probe alters the offset times involved in performing wave separation, however these times can be calculated using the relevant travel paths and sound speeds in the bar, discussed in section III A.

The free face of the striker bar was not chosen as either the striker bar would have to leave the barrel by a significant distance to allow it to be properly probed, overcomplicating system alignment, or significant changes to a typical Hopkinson bar set-up would have to be made in order to allow the fibre to enter the barrel at a shallow angle. The method we propose requires no bar or barrel modifications to implement the PDV.

\section{METHOD OF ANALYSIS}

\section{A. Internal Reflection in the Striker Bar}

Due to the nature and timescale of the unloading in the striker bar during direct impact, measurements of the sample face velocity will usually be superimposed with the tensile wave reflections of compressional waves returning from the free end of the striker bar. Separation of these waves becomes further complicated by the bulk velocity of the wave medium with reference to the probe. The velocity measured by the probe is the combination of three separate components,

$$
V_{\text {probe }}=V_{\text {bulk }}+V_{\text {compressive }}+V_{\text {tensile }} .
$$

In order to obtain the force and velocity history of the sample face, these profiles must be correctly separated and time shifted. Separation is possible as the initial bulk velocity is measurable, and the initial tensile velocity is zero.

Figure 5 depicts the complete path of one wave in the striker. The compression wave is formed at the sample, (a), at $t_{1}$ and passes by the measurement probe (b) at $t_{2}$. It then reaches the back surface (c) where the free end boundary condition forces it to be reflected into a tensile wave. The tensile wave passes back past the measurement probe at $(\mathrm{d})$ at a time $t_{3}$ and contributes a further change in the velocity at that point, equal to that the compression wave at (b). After passing as a reflected wave, the tensile wave returns to the sample at (e) after which any effects of the wave can be considered as part of the motion at the sample face, and do not require separating and correcting.

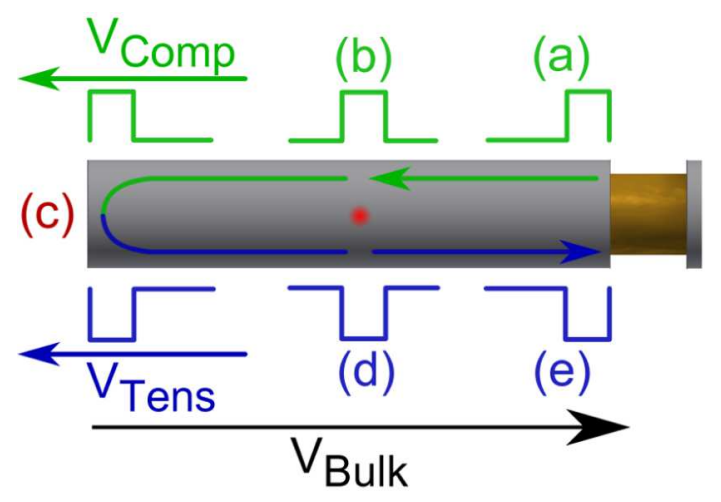

FIG. 5. The complete path of a compression wave from the sample to the free face and back as a tensile wave, passing the measurement probe each way. Also labeled outside the bar are the directions of the contributions to local particle velocity from each pulse relative to the bulk velocity. Note that the tensile wave further decelerates the striker bar due to the inversion of both wave type and travel direction.

The initial velocity at the struck face of the specimen is the signal in the absence of the reflected trace, as that is the only velocity not to originate from the sample. The 


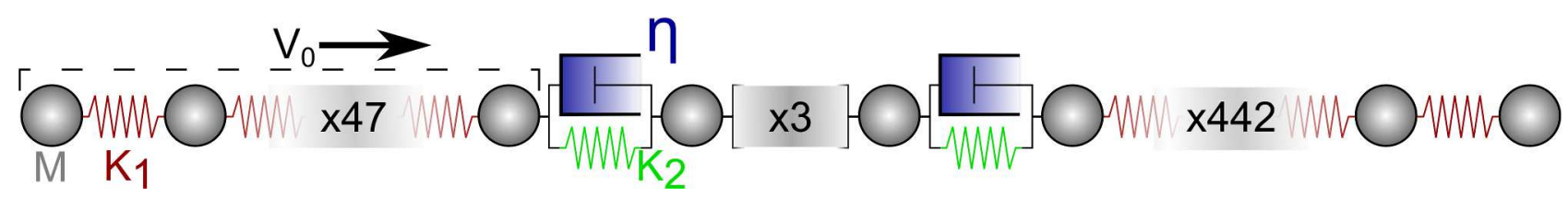

FIG. 6. A sketch of the simulation setup. A 50 mass elastic striker with initial velocity $V_{0}=100 \mathrm{~ms}^{-1}$ impacts a 5 mass viscoelastic sample connected to another 445 mass output bar. The sample uses a Kelvin-Vöigt parallel spring and dashpot model. Tensile forces were turned off at the interface atoms.

corrected trace is then given by

$$
V_{\text {st }}\left(t_{1}\right)=V_{\text {probe }}\left(t_{2}\right)-V_{\text {tensile }}\left(t_{2}\right),
$$

where $V_{s t}$ is the velocity of the struck face of the sample, and timeshifting from $t_{2}$ to $t_{1}$ is applied as discussed above. Given perfect reflections, the inversion of both wave type and travel direction at the striker free end means the velocity change due to the tensile component is the same as that of the equivalent earlier compressive component,

$$
V_{\text {tensile }}\left(t_{2}\right)=V_{\text {compressive }}\left(t_{3}\right) .
$$

\section{B. Trace Recovery Simulations}

We generally wish to obtain $V_{s t}$ and $V_{c o m p}$, to enable the deformation and force histories to be extracted. Whilst simulations are not required for any of the experimental analysis, to illustrate and confirm the correction procedure, we used a simple $1 \mathrm{D}$ simulation shown schematically in Figure 6. A 50 long chain of unit masses were connected by springs of equal spring constant, $K_{1}$, to represent an elastic striker bar. These were set to an initial speed, $V_{0}$, of $100 \mathrm{~ms}^{-1}$ and connected to a 5 long chain of equal masses with restoring force dictated by a Kelvin-Voigt viscoelastic material model to representing the sample. For a mass in the middle of the sample the force experienced took the functional form

$$
\begin{aligned}
& F_{i}(t)=K_{2}\left[X_{i-1}(t)+X_{I+1}(t)-2 X_{i}(t)\right] \\
&+\eta\left[V_{i-1}(t)+V_{I+1}(t)-2 V_{i}(t)\right]
\end{aligned}
$$

where $X_{i}(\mathrm{t})$ and $V(t)_{i}$ are the position and velocity of mass $i$ as a function of time. After the sample chain was a 445 long chain of unit masses, of spring strength equal to the striker bar, modelling the output bar and preventing the output wave reflecting back during the time scale of the simulation.

Values of $K_{2}$ and $\eta$ were chosen to provide a complex velocity history, with steps between complete transits of the compressional wave, not always observed in real samples. These extra steps provide more complex features to check the fidelity of our reflection correction procedure.

Velocity histories were obtained by extracting the average velocity of the mass of interest and two masses either side, to simulate the broadness of a real PDV probe and remove high frequency oscillations of individual masses about their net motion. The probes were placed with their centres in the middle of the striker bar, and on the striker side face of the sample. It should be emphasised at this point that such a measurement of the struck face of the sample is not possible in 2D as Saint-Venant's principle shows surface measurements at this point unrepresentative of the bulk. The results of these two extractions are shown in Figure 7, and discussed in the next section. The probe measurement (solid line) has been time shifted onto the sample face measurement (dotted line).

\section{Reflection Correction Procedure}

For the purpose of discussing the correction procedure simply, we define a dimensionless time, $\tau$, such that one unit is the time taken for a sound wave to travel one length of the striker bar,

$$
\tau_{i}=\frac{C_{s t r}}{L_{s t r}} t_{i} .
$$

where $\mathrm{C}_{s t r}$ is the sound speed and $L_{s t r}$ is the length of the striker bar. For a probe at the midpoint of the striker bar, the dimensionless time between $\tau_{1}$ and $\tau_{2}$ is 0.5 and between $\tau_{2}$ and $\tau_{3}$ is $1\left(t_{2}>t_{1}>t_{3}\right)$. For a probe on the free end of the bar $\tau_{3}=\tau_{2}=\tau_{1}+1$.

Due to the reflections taking one unit of dimensionless time to return, we know that for $\tau<1$ our trace does not require correction, this is depicted in Region I of Figure 7. In this region, given the initial velocity from either light gate or pre-impact PDV measurements, we can extract the compression velocity from the difference between the initial and measured velocity, as shown by the blue down pointing arrows in Region I.

As we now know the tensile velocity which returns to the probe, we can correct the probe trace by subtracting that velocity from the probe measurement, as shown by the same blue arrows from Region I, inverted one unit of $\tau$ later in region II. At this point the trace in Region II is correct, and the process can be repeated to correct Region III. However during this time the bulk velocity of the striker bar is likely to have decreased due to a deceleration step making a complete transit of the bar. The bulk velocity for any region is that of the most recently completed velocity step before that region. In 


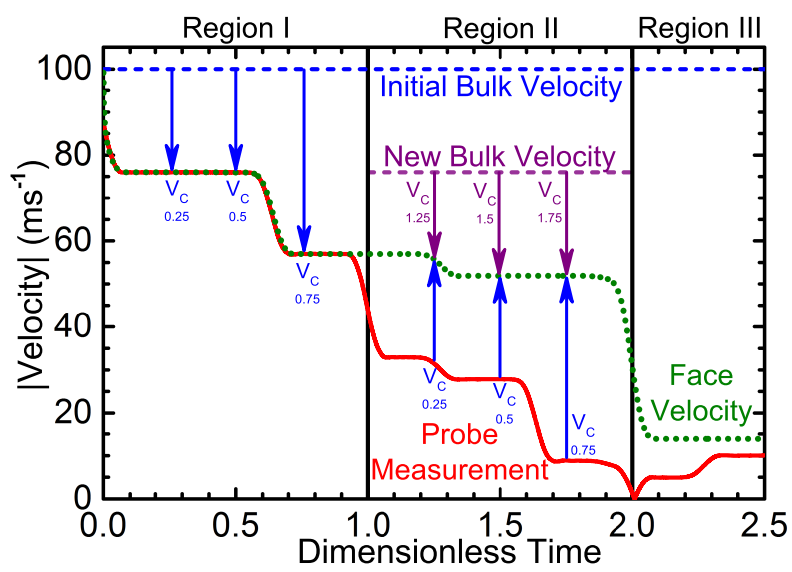

FIG. 7. A graph depicting the stages of the reflection correction procedure to convert the probe measurement (solid line) to the true striker sample face velocity (dotted line), outlined in the bulk text. At $\tau=2$ the striker bar velocity passes through zero and becomes negative, however it appears positive as PDV measurements are direction insensitive.

Region I we observe two velocities, approximately 76 and $59 \mathrm{~ms}^{-1}$, whilst the $59 \mathrm{~ms}^{-1}$ is the more recently reported velocity, as its step continues into Region II it cannot have completed decelerating the entire bar, making the new bulk velocity $76 \mathrm{~ms}^{-1}$ as labelled in Figure 7.

Time shifting for the compressional waves can be performed more reliably in the case of striker reflections than reflections in the input bar in an SHPB, as the shifting takes place between parts of the same monotonically decreasing wave; any incorrect shifting produces obvious spikes in the recovered velocity.

\section{Eulerian vs Lagrangian Measurements}

As the probes remain fixed whilst the bars move during the experiment, they perform Eulerian measurements rather than the Lagrangian measurements made by bar mounted gauges. This creates an effect similar to a Doppler shift, where the measurements from a face approaching the measurement probe occur more often than a stationary one as the distance the time shift back from the probe to the sample face is constantly decreasing. All corrections regarding these effects are of the order $\mathrm{V}(\mathrm{t}) / C_{b a r}$, so for typical bar impact speeds of $1-10 \mathrm{~m} \mathrm{~s}^{-1}$ the correction is small. However at the target rates for a DIHB, where velocities are closer to $100 \mathrm{~m} \mathrm{~s}^{-1}$, the corrections are of the $1 \%$ order of magnitude.

This effect can be corrected for both the striker and output bar by updating the probe positions at each time step in the analysis. If the probe measures a velocity $\mathrm{V}(\mathrm{t})$ at time $t$, then the probe position on the bar changes by approximately $-\mathrm{V}(\mathrm{t}) \Delta t$ between two measurements taken $\Delta t$ apart. The position is updated and allows for recalculation of the relevant distances for the purposes of time shifting and determining when reflected waves return.

Output bar measurements can be corrected simply by time shifting the initial point and by altering the time period between measurements to account for the decreasing distance between the sample face and the probe:

$$
\Delta t^{\prime}=\Delta t\left(1-\frac{V(t)}{C}\right)
$$

where $\Delta \mathrm{t}$ is the time between two measurements at the probe and $\Delta t^{\prime}$ is the time between two measurements at the sample surface. As the sample face moves towards the probe the true time period between measurements is faster than the sampling rate of the probe.

For the striker bar, the varying position not only decreases the frequency of sampling of the sample face relative to the actual rate at which the probe samples in the form

$$
\Delta t^{\prime}=\Delta t\left(1+\frac{V(t)}{C}\right)
$$

but also decreases the time taken for reflected waves to return. The decreased time between passing the probe for the first time and returning as a tensile wave can simply be found by updating the position of the probe, and given that the motion is small compared to the sound speed of the bars, the return time can be closely approximated as twice the distance between the probe and the free end over the bar sound speed.

If sampling is performed at the free end of the striker it does not require any Eulerian corrections as the probe remains at the same point on the bar during deformation. However the alteration of the typical bar system setup required to allow a fibre access to the free end of the striker bar are more complex than updating the striker position during analysis.

\section{E. Sample Deformation Calculation}

Once measurements have been made of the front, or striker impact face, $V_{s t}$, and the back, or output bar face, $V_{o p}$, the sample length as a function of time is determined by the difference between these two velocities

$$
\frac{\partial L(t)}{\partial t}=V_{o p}-V_{s t}
$$

and

$$
\varepsilon(t)=\ln \left(\frac{L(0)}{L(t)}\right),
$$

where compressive strains are considered positive. The stress history of the sample is measured as per a classic DIPB test, using the elastic relation

$$
\sigma(t)=V_{o p}(t) Z_{o p} \frac{A_{o p}}{A_{s a}(t)},
$$


where $\mathrm{Z}_{o p}$ is the acoustic impedance of the output bar and $\mathrm{A}_{o p}$ and $\mathrm{A}_{s a}$ are the cross sectional areas of the output bar and sample respectively. For fully solid samples the area is calculated using the approximation of constant volume during plastic deformation

$$
L(t) A_{s a}(t)=L(0) A_{s a}(0) .
$$

\section{F. Force Measurements and Equilibrium}

The force at the output face can be found directly from the measured velocity

$$
F_{o p}(t)=V_{o p}(t) Z_{o p} A_{o p},
$$

the same relationship holds true for the striker bar. However, the only velocity component due to the force from the sample is the compressive wave

$$
F_{\text {st }}(t)=Z_{\text {str }} A_{\text {str }} V_{\text {compressive }}(t) .
$$

These measurements allow force equilibrium analysis to be performed in exactly the same way as classic split bar tests. It should be noted that bars of equal acoustic impedance and area, a compressional velocity comparison is equivalent to a force comparison.

\section{EXPERIMENTAL RESULTS \& DISCUSSION}

An array of samples were tested at equal striker velocities in both SHPB and DIHB arrangements. SHPB velocity traces from the surface were analysed using standard three-wave methods ${ }^{9}$. DIHB data was analysed using the two-wave analysis described in Section III, and traditional one-wave analysis ${ }^{3}$ where for matching material and diameter bars, as in our experimental setup, the struck face velocity is approximated by

$$
V_{s t}(t)=V_{0}-V_{o p}(t)
$$

where $V_{0}$ is the firing velocity of the striker.

\section{A. Fully Dense Metals}

Sample disks of $1 \mathrm{~mm}$ thickness and $3 \mathrm{~mm}$ diameter were made from a $99.9 \%$ pure aluminium and a commercial Dural alloy. The stress-strain relationships obtained using these methods are depicted in Figures 8 and 9 . Figure 8 is cropped to a true strain of 0.08 to allow clearer comparison between the DIHB methods and the SHPB results. Higher strain curves for both DIHB methods in the case of pure aluminium are depicted in Figure 9.

At true strains lower than 0.05 , two-wave analysis of the DIHB data is a closer match to the SHPB data. This is likely due to the one-wave DIHB measurement

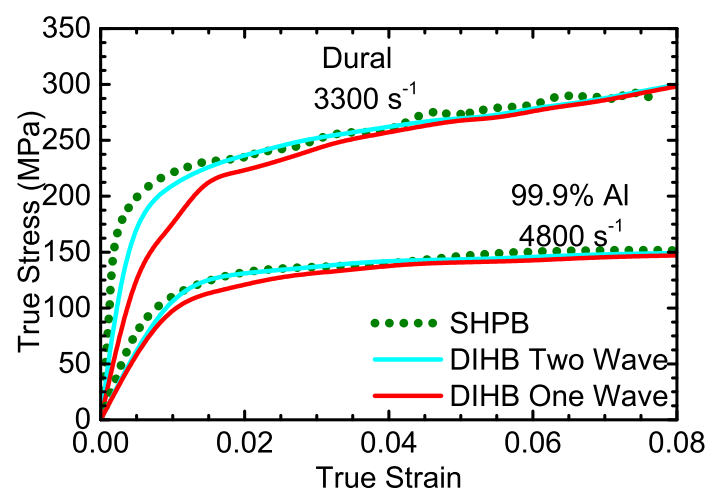

FIG. 8. The stress-strain relationships for equal striker velocity tests in SHPB and DIHB setups. Two-wave analysis of the DIHB data is a better match to SHPB data early on. One-wave measurements reach similar accuracy after a true strain of approximately 0.05 .

incorrectly assuming force equilibrium during ring up in the sample. Figure 10 depicts the forces on the sample faces in the $99.9 \%$ aluminium DIHB test. At approximately $10 \mu$ s after the initial rise, yield can be seen triggering the onset of force equilibrium between the faces. This time corresponds to a true strain of approximately 0.05 at a strain rate of $4800 \mathrm{~s}^{-1}$, in agreement with the initial observation.

After the initial $10 \mu s$ of Figure 10 the forces quickly equilibrate as sample yields. At equilibrium the force at the struck face oscillates about the smoother force trace of the output face, similarly to that of the input face of an SHPB. However in the DIHB the oscillations are of lower frequency. The struck face force oscillations are likely to be dominated by the return of the compression pulse after a complete transit of the striker bar, involving higher forces and longer time scales than ringing within the sample itself. Striker bar transits explain the typical time period between the oscillations; in Figure 10 the oscillations appear to be approximately every $50 \mu \mathrm{s}$ which matches one length of the $25 \mathrm{~cm}$ long

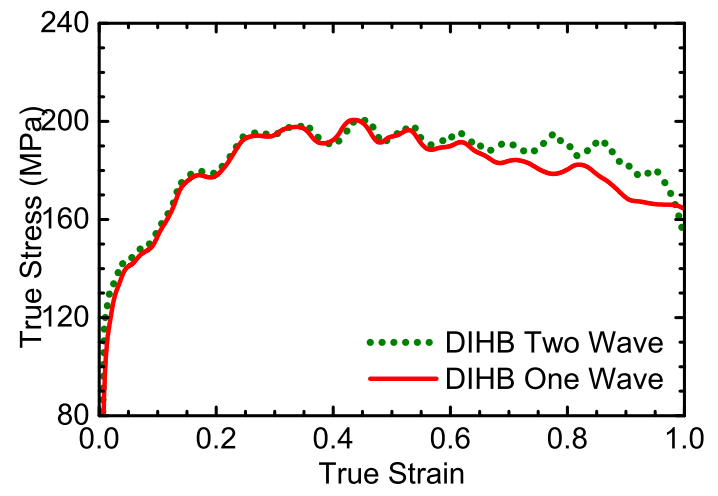

FIG. 9. The higher strain trace for $99.9 \%$ pure $\mathrm{Al}$ from Figure 8. The one wave trace softens notably above 0.5 true strain. 


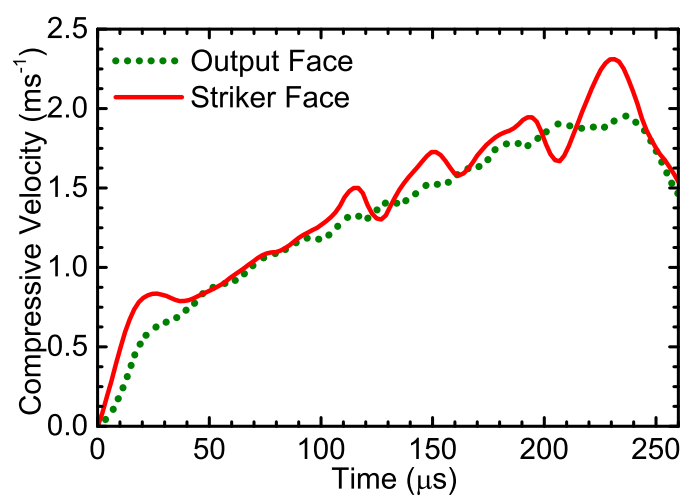

FIG. 10. The compressional wave velocies leaving the sample through the striker bar and output bar for one pure aluminium sample. These are proportional to the forces on the sample faces, shown in equations 18 and 19.

striker bar with a sound speed of approximately 5.1 $\mathrm{km} \mathrm{s}^{-1}$. Errors also arise due to imperfect corrections which are most notable at the steeper regions of the velocity steps, these grow with successive applications of the correction procedure but are confined only to the transitions between steps, and only occur late in the trace when force equilibrium has been verified.

Of note is the linear, or pre-yield region of the stress-strain curves which extends to approximately 0.01 true strain for both specimens. In non-dynamic loading,this would correspond to a differing elastic modulus of a sample. However, as the samples are loaded dynamically and are not yet in force equilibrium, a steeper curve corresponds to better transmission of the stress wave at the input and output boundaries between the sample and the bars. In the results provided, the SHPB gave a faster rise to equilibrium as the impedance was better matched due to the $12.5 \mathrm{~mm}$ bars used in the SHPB compared to the $25 \mathrm{~mm}$ bars in the DIHB ${ }^{13}$.

For strains greater than 0.5 the one-wave trace is seen to consistently decrease below the two-wave trace,

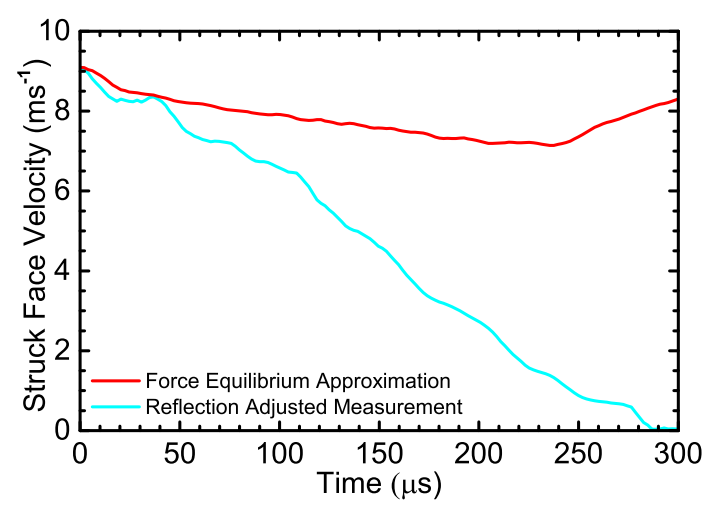

FIG. 11. The values of $V_{s t}$ for the pure aluminium sample in Figure 10 generated by reflection corrected measurements and using approximation 20. shown in Figure 9. By this point the bulk velocity of the striker bar has been reduced by multiple passes of decelerating waves. In one-wave analysis the striker bulk velocity is usually assumed constant, as in equation 20 . This leads to an over-estimation of strain and therefore sample area due to volume conservation, leading to an underestimate of the flow stress in equation 16. The error is explicitly shown in Figure 11 where the approximate and measured velocities are compared. Notably there is an upturn in the approximation trace at $250 \mu \mathrm{s}$ which causes the strain measurement after that point to diverge. Traces generated using the the measured trace remain well behaved even during the unloading of the specimen.

\section{B. Foams \& Cellular Materials}

Disk samples of $3 \mathrm{~mm}$ thickness and $6 \mathrm{~mm}$ diameter of an aluminium foam were tested and analysed using the same methods as in section IV A. Magnesium bars of $12.5 \mathrm{~mm}$ diameter were used due to low expected sample strength. The resultant traces are displayed in Figure 12.

The results show the one-wave method projecting the deformation profile onto higher strains than the two wave measurement, the same effects as were observed for fully dense metals. Also of note is a shallow rise present in the first $5 \%$ engineering strain of the one wave case, but not in the two wave measurement.

In foams and cellular materials, collapse of the internal structure leads to the localisation of deformation ${ }^{14}$ and alters localised properties such as the mechanical impedance. Localised processes affect the relationship between the forces on the sample faces in a way that is not only dependant on the sample, but is also time varying and dependant on the conditions and type of impact.

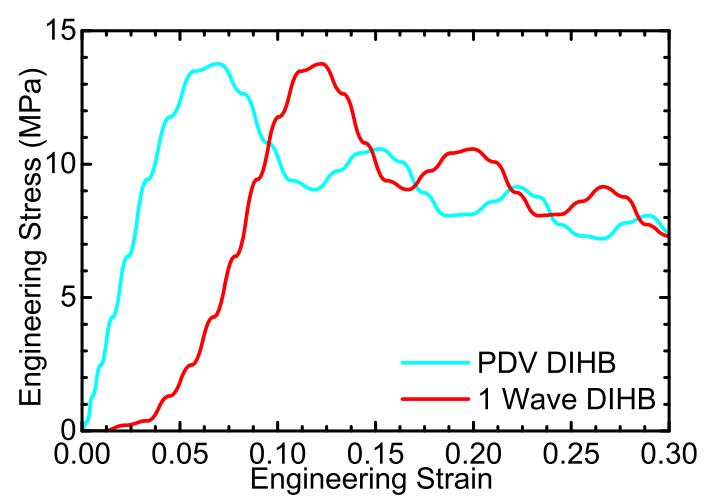

FIG. 12. The resultant stress-strain traces for a DIHB sample analysed using both 1 and 2 wave methods. Due to localisation and compaction true stress and true strain measurements are not as physically meaningful as for other materials, engineering values have been calculated instead. The engineering strain rate in the experiments performed was approximately $4500 \mathrm{~s}^{-1}$ 
Comparative SHPB data is not presented here. SHPB tests may provide a different loading profile in foams as the elastic ring up allows the deformation to be less localised than in a DIHB. The gradual rise of the applied stress, due to input dispersion and incomplete transmission at the input boundary, allows the force to propagate throughout the sample, causing widespread elastic and potentially non cell collapsing plastic deformation to occur before the force reaches a high enough magnitude to cause cells to collapse ${ }^{15}$. In the DIHB the sample is responsible for the force at the impacted face, which will correspond to the highest force generating mechanism appropriate for the rate of deformation. At the impact speeds available in bar tests, thes mechanism is most likely cell collapse. Thus, SHPB and DIHB tests with foam like materials may provide different stress strain histories for equivalent strain rates in the two tests, due to the different underlying microstructural processes they are most likely to initiate. This prevents any differences between the results of equivalent SHPB and DIHB experiments being conclusively attributed to the method of measurement. This issue is outside the focus of this paper.

\section{Polymers \& Slow Sound Speed Materials}

PTFE samples of $2 \mathrm{~mm}$ thickness and $9 \mathrm{~mm}$ diameter were placed in DIHB and SHPB arrangements and impacted with equal striker velocities, and analysed using both one and two wave DIHB analysis. The one of the results from each arrangement can be seen in Figure 13. Both experiments were performed using $12.5 \mathrm{~mm}$ Dural bars.

The results show an erroneous softening exclusively in the one wave analysis, the two wave DIHB and the SHPB tests are in good agreement. This is the same relationship as observed in Figure 8 however to a greater extent and approximately $10 \%$ of the SHPB value.

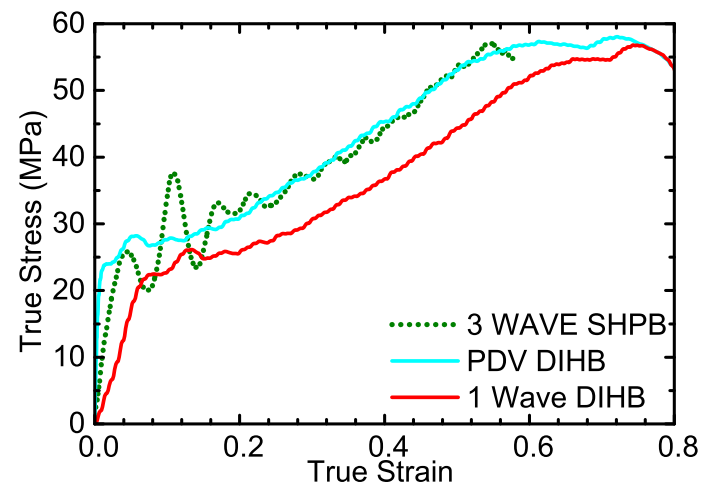

FIG. 13. The final stress strain traces for fully dense polyethylene using three wave SHPB, one wave DIHB and two wave PDV analysis. The true strain rates in these experiments were approximately $7600 \mathrm{~s}^{-1}$.
Polymers are capable of reaching force equilibrium, however due to the relatively slow sound speeds, poor impedance matching with typical bar materials and the lack of any on-yield equilibration mechanism makes the time taken much longer than materials such as metals.

During this long ring-up time the velocity of the impacted face of the sample is over-estimated, as it was in previous sections. The larger timescale means any strain calculated using approximation 20 is incorrect by a larger amount and thus so is the area predicted using volume conservation.

Unlike in section IV A, in these polymer experiments, the impedance match between the sample and the bars is the same, as the DIHB and SHPB bars were of equal diameter and of the same material. Here, the rise of the sample to equilibrium is steeper in the DIHB case, implying a faster rise to force equilibrium. The faster rise is likely due to the force effectively originating from the sample in the direct impact scenario, greatly relieving the boundary transmission problem at the input face, leaving only the output face to delay equilibrium.

\section{CONCLUSIONS}

We have presented a method for directly measuring the velocities and forces at the impacted face of a specimen in a DIHB experiment. Analysis of the data was demonstrated including the recovery of the sample face trace in the presence of free end striker reflections. Experimental data comparing the method to current approximations was presented with reference to equivalent SHPB data. Direct measurements were shown to more closely correspond to SHPB measurements at low strains and to not suffer from the same high strain inaccuracies as assuming constant striker inertia. Our method was demonstrated improves the viability of DIHB measurements for samples which do not quickly reach force equilibrium, including polymers, foams and cellular materials, with minimal alteration of the bar system itself. We expect the technique to be of wide applicability, potentially allowing access to previously untested strain regimes.

\section{ACKNOWLEDGMENTS}

This research was supported by grants from QinetiQ and the Engineering and Physical Sciences Research Council, as per EPSRC open data requirements, the data presented in this article can be found at https://www.repository.cam.ac.uk/handle/1810/252435

The authors would like to thank P.J. Gould and P.D. Church for their interest. 
${ }^{1} \mathrm{H}$ Kolsky, Proceedings of the Physical Society B 62, 676-700 (1949)

${ }^{2}$ M Meyers, Dynamic Behavior of Materials 305-310, (1994)

${ }^{3}$ D Gorham, P Pope, J Field, Proceedings of the Royal Society A 438, 153170 (1992)

${ }^{4} \mathrm{H}$ Couque, Philosophical Transactions of the Royal Society A, 372, 20130218 (2015)

${ }^{5} \mathrm{~A}$ Love, A treatise on the mathematical theory of elasticity (Cambridge University Press, 1927) 257-258

${ }^{6} \mathrm{D}$ Gorham, Institute of Physics Conference Series 47, 16-24 (1980)

${ }^{7} \mathrm{D}$ Gorham, Proceedings of the 13th International Congress on High Speed Photography and Photonics, Tokyo, 1978
${ }^{8} \mathrm{R}$ Govender, Unpublished Presentation at DYMAT Hopkinson Centenary Conference (2014)

${ }^{9} \mathrm{C}$ Avinadav, $\mathrm{Y}$ Ashuach, $\mathrm{R}$ Kreif, Review of Scientific Instruments 82, 073908 (2011)

${ }^{10}$ S Walley, J Field, P Pope, N Safford, Philosophical Transactions of the Royal Society of London, Series A 328, 1597 (1989)

${ }^{11} \mathrm{O}$ Strand, D Goosman, C Martinez, T Whitworth, W Kuhlow, Review of Scientific Instruments 77, 083108 (2006)

${ }^{12}$ D Casem, M Zellner, Experimental Mechanics 53, 14671473 (2013)

${ }^{13} \mathrm{~J}$ Achenbach, Wave Propagation in Elastic Solids (Elsevier Science Publishers, 1973)

${ }^{14}$ Yang et al. Material Science and Engineering A 560,734, 2013

${ }^{15}$ Cady et al. Materials Science and Engineering A 525, 1, 2009 